\title{
Proteomic analysis of Staphylococcus aureus biofilm cells grown under physiologically relevant fluid shear stress conditions
}

\author{
Nazrul Islam ${ }^{3^{*}}$, Yonghyun Kim² ${ }^{*}$, Julia M Ross ${ }^{1}$ and Mark R Marten ${ }^{1}$
}

\begin{abstract}
Background: The biofilm forming bacterium Staphylococcus aureus is responsible for maladies ranging from severe skin infection to major diseases such as bacteremia, endocarditis and osteomyelitis. A flow displacement system was used to grow S. aureus biofilms in four physiologically relevant fluid shear rates $\left(50,100,500\right.$ and $1000 \mathrm{~s}^{-1}$ ) to identify proteins that are associated with biofilm.

Results: Global protein expressions from the membrane and cytosolic fractions of $S$. aureus biofilm cells grown under the above shear rate conditions are reported. Sixteen proteins in the membrane-enriched fraction and eight proteins in the cytosolic fraction showed significantly altered expression $(p<0.05)$ under increasing fluid shear. These 24 proteins were identified using nano-LC-ESI-MS/MS. They were found to be associated with various metabolic functions such as glycolysis / TCA pathways, protein synthesis and stress tolerance. Increased fluid shear stress did not influence the expression of two important surface binding proteins: fibronectin-binding and collagen-binding proteins.

Conclusions: The reported data suggest that while the general metabolic function of the sessile bacteria is minimal under high fluid shear stress conditions, they seem to retain the binding capacity to initiate new infections.
\end{abstract}

Keywords: Biofilm, Staphylococcus aureus, Flow chamber, Shear stress, Proteomics

\section{Background}

A biofilm is an aggregate of bacterial microcolonies adherent to each other and to biotic or abiotic surfaces, embedded in an extracellular matrix produced by the sessile bacteria composing the microcolonies. Biofilm has been found in $65-80 \%$ of the bacterial infections, and is considered refractory to host defenses in antibiotic therapy [1]. Among the biofilm forming bacteria, Staphylococcus aureus is responsible for severe skin infections to such major diseases as bacteremia, endocarditis and osteomyelitis. Under suitable conditions, S. aureus causes serious complications in devices like implants and catheters by producing biofilms on them $[2,3]$. Treatment of such infections becomes even more challenging given that several S. aureus strains show resistance to multiple antibiotics

\footnotetext{
*Correspondence: nazrul@umd.edu; ykim@eng.ua.edu

${ }^{3}$ Current address: Department of Plant Sciences, University of Maryland, College Park, MD 20742, USA

${ }^{2}$ Department of Chemical and Biological Engineering, The University of Alabama, Tuscaloosa, AL 35487, USA

Full list of author information is available at the end of the article
}

(e.g., methicilin and vancomycin). Donlan and Costerton [4] postulated three mechanisms responsible for antibiotic resistance: (i) delayed penetration of the antimicrobial agent through the biofilm matrix, (ii) altered growth rate of biofilm organisms, and (iii) other physiological changes due to the biofilm mode of growth. Growth rate and physiological changes are considered the major causes of resistance to antibiotics. Characterizing bacterial biofilm architecture and stability in their favorable growth conditions would help elucidate how these bacteria survive during their processes of biofilm formation.

In general, increased antimicrobial tolerance is related to the biofilm physiology and architecture [5]. Architecture depends on (i) the development of an extracellular matrix composed of proteins, DNAs, minerals and/or polysaccharides secreted by the sessile cells and (ii) the shear environment in which matrix development occurs [4]. The stability of the structure lies in its viscoelastic properties and its related ability to withstand mechanical cleansing forces (e.g., fluid shear stress) [6]. A number of previous studies investigated the mechanism of 
adhesion and erosion of $S$. aureus biofilm cells under physiologically relevant fluid shear conditions [7-13]. The latter study demonstrated that platelets (thrombocytes) adhere to the collagen (fibrous structured proteins in blood vessels) of the vascular sub-endothelium in distinct patterns. Shear rates of $\leq 800 \mathrm{~s}^{-1}$ were demonstrated to allow better adhesion than the higher shear rates.

In addition to the above, several studies focused on the protein profiles of $S$. aureus biofilm cells under different growth conditions [14-16]. Higher levels of surface proteins, especially the fibrinogen-binding proteins, were observed in biofilm cells when compared to planktonic cells [17]. Similarly, several other studies investigated protein profile of $S$. aureus biofilm cells under different growth conditions most of which employed either shake flasks or nutrient-rich agar plates to produce biofilm [14-16]. Nonetheless, use of shake flasks or agar plates does not seem to provide appropriate environment for the physiologically relevant biofilm studies for several reasons $[17,18]$. First, it exposes the bacteria to nutritionally rich media. Second, biofilm grown in shake flasks encounters high osmolarity, oxygen limitation and a relatively high cell density that together influence gene expression in S. aureus [19-21]. Third, shake flasks do not mimic the in-vivo environment where Staphylococci grow as biofilm under fluid shear.

Investigations discussed thus far provided significant information to the molecular mechanisms of biofilm formation, erosion and dissemination. However, little is known how the overall protein profiles in S. aureus biofilm cells are influenced under different fluid shear rates.
It is hypothesized that fluid shear stress influences gene regulatory networks controlling protein expression and thereby contributing to bacterial adhesion/survival during infection. In this manuscript, a proteomic approach to study the expression of membrane and cytosolic protein fractions in biofilm cells grown under physiologically relevant fluid shears is reported. The protein expression profiles of biofilm cells under different shear conditions provide novel insights into the cellular processes related to bacterial adhesions/survival during infection in vivo.

\section{Results and discussion}

\section{2-DE profile, protein identification and classification}

For the comparison of protein profiles under different shear rates, a two dimensional electrophoresis (2-DE) profile was generated using membrane protein fractions from cells grown in shake flasks for two hours. It is known that most of the proteins associated with bacterial adhesion to the substratum are located in the membrane fraction. Of the $200 \pm 5$ protein spots resolved in four biological replicate gels, 64 were confidently identified using nano-LCMS/MS (Figure 1 and Table 1). The remaining 136 protein spots could not be identified due to their low abundance and were below the limit of identification. As shown in Table 1, most of the proteins were identified with high XC score and sequence coverage. Using PSORT [22] and TMpred [23], the subcellular location of 16 proteins were identified as membrane and 20 proteins as transmembrane. Twenty-six proteins were also predicted to be of cytoplasmic origin, which may have originated from lysed cells. The exact reason for the contamination of proteins

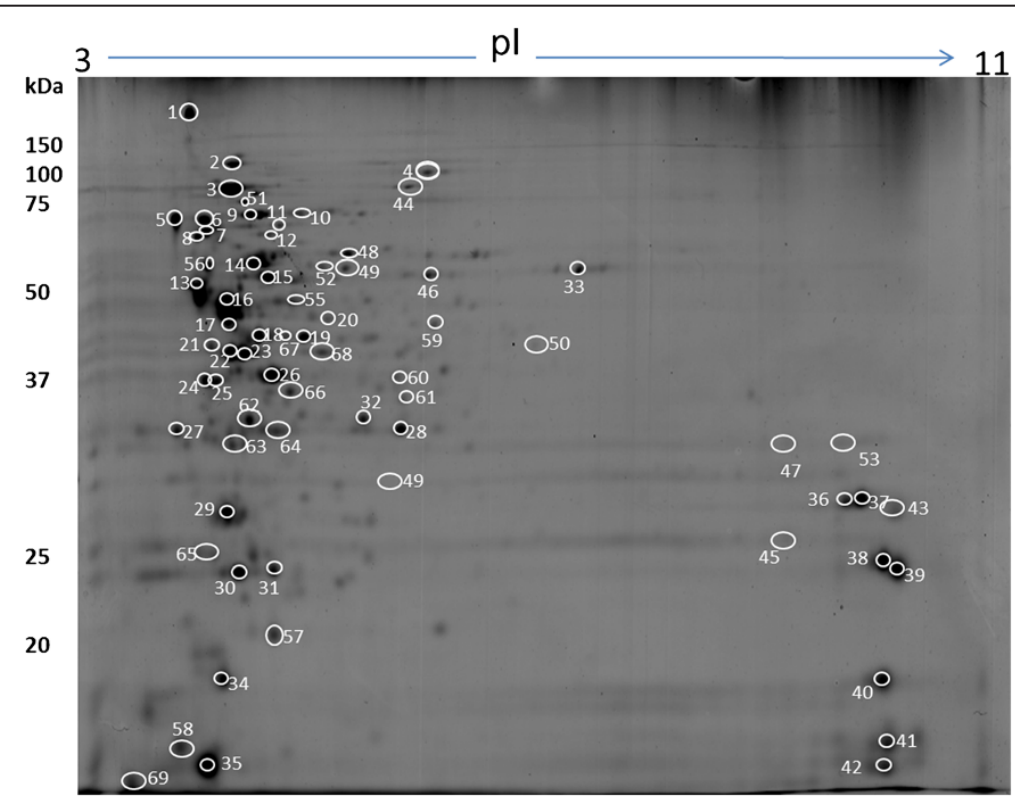

Figure 1 A 2-DE gel image of Staphylococcus aureus. Membrane protein fraction of bacteria grown in shake flasks for 2 hrs was extracted by digesting with lysostaphin in presence of raffinose. Numbered proteins were identified by nano-LC ESI MS and shown in Table 1. 
Table 1 Proteins shown in the reference map were identified with a linear ion trap nano-LC-ESI-MS/MS

\begin{tabular}{|c|c|c|c|c|c|c|c|}
\hline Spot ID & Protein name & Functional category & XC-score & $\mathrm{pl} / \mathrm{Mr}$ & Cov (\%) & Accession number & Location \\
\hline 1 & Fibronectin-binding protein & Virulence mechanism & 150 & $4.4 / 114$ & 18 & gi:15925493 & Membrane \\
\hline 2 & Aconitate hydratase & Carbohydrate metabolism & 396 & $4.7 / 98$ & 51 & gi:15924340 & transmembrane \\
\hline 3 & Elongation factor $\mathrm{G}$ & Transcription and replication & 548 & $4.6 / 77$ & 49 & gi:15923537 & transmembrane \\
\hline 4 & Collagen Binding Protein (Cna) & Virulence mechanism & 90 & $5.8 / 133$ & 11 & gi:21284341 & Membrane \\
\hline 5 & Trigger factor & Virulence mechanism & 140 & $4.2 / 49$ & 36 & gi:15924665 & Cytosolic \\
\hline 6 & DnaK protein & Stress protein & 348 & $4.5 / 66$ & 67 & gi:15924570 & Membrane \\
\hline 7 & DnaK protein & Stress protein & 218 & $4.5 / 66$ & 51 & gi:15924570 & Membrane \\
\hline 8 & PEP-protein phosphatase & Carbohydrate metabolism & 60 & $4.5 / 63$ & 20 & gi:15926670 & Membrane \\
\hline 9 & PEP-protein phosphatase & Carbohydrate metabolism & 30 & $4.5 / 63$ & 18 & gi:15926670 & Membrane \\
\hline 10 & Pyruvate kinase & Carbohydrate metabolism & 150 & $4.1 / 63$ & 25 & gi:15924687 & transmembrane \\
\hline 11 & Prolyl-tRNA synthetase & Cell division & 228 & $4.9 / 64$ & 54 & gi:15924253 & transmembrane \\
\hline 12 & Prolyl-tRNA synthetase & Cell division & 58 & $4.9 / 64$ & 13 & gi:15924253 & transmembrane \\
\hline 13 & 305 ribosomal protein & Protein synthesis & 130 & $4.4 / 43$ & 37 & gi:15924466 & transmembrane \\
\hline 14 & Glutamyl- amidotransferase & Aminio acid metabolism & 84 & $4.9 / 53$ & 25 & gi:21283570 & transmembrane \\
\hline 15 & Glycyl-tRNA synthetase & Cell division & 90 & $4.9 / 54$ & 20 & gi:15924555 & Cytosolic \\
\hline 16,17 & Elongation factor Tu & Virulence mechanism & 214 & $4.6 / 43$ & 60 & gi:15923538 & Membrane \\
\hline 18 & Pyruvate dehydrogenase & Virulence mechanism & 180 & $4.8 / 41$ & 48 & gi:15924083 & Membrane \\
\hline 19 & Phosphoglycerate kinase & Carbohydrate metabolism & 120 & $5.1 / 42$ & 42 & gi:15923763 & transmembrane \\
\hline 20 & Coenzyme A ligase & Protein synthesis & 140 & $5.0 / 43$ & 42 & gi:21282234 & transmembrane \\
\hline 21 & DNA-directed RNA polymerase & Transcription and replication & 110 & $4.5 / 35$ & 38 & gi:15925214 & Cytosolic \\
\hline 22 & Glyceraldehyde-3-PDH & Carbohydrate metabolism & 108 & $4.8 / 36$ & 44 & gi:15923762 & Cytosolic \\
\hline 23 & Coenzyme A synthase & Carbohydrate metabolism & 30 & $4.80 / 43$ & 20 & gi:49484747 & transmembrane \\
\hline 24 & Pyrophosphatase & Carbohydrate metabolism & 30 & $4.5 / 34$ & 15 & gi:15924909 & Membrane \\
\hline 25 & Phosphotransacetylase & Others & 102 & $4.6 / 35$ & 55 & gi:15923578 & Cytosolic \\
\hline 26 & Elongation factor Ts & Transcription and replication & 190 & $5.0 / 32$ & 58 & gi:15924247 & Cytosolic \\
\hline 27 & General stress protein Ctc & Stress protein & 50 & $4.2 / 24$ & 29 & gi:15923491 & Cytosolic \\
\hline 28 & 305 ribosomal protein & Protein synthesis & 106 & $5.3 / 30$ & 30 & gi:15924035 & transmembrane \\
\hline 29 & Triosephosphate isomerase & Carbohydrate metabolism & 58 & $4.7 / 27$ & 32 & gi:15923764 & Cytosolic \\
\hline 31 & Superoxide dimutase & Stress protein & 80 & $5.0 / 23$ & 35 & gi:15924543 & Cytosolic \\
\hline 32 & Cysteine synthase & Carbohydrate metabolism & 60 & $5.2 / 33$ & 24 & gi:15923503 & transmembrane \\
\hline 33 & Malate:quinone oxidoreductase & Carbohydrate metabolism & 220 & $6.1 / 56$ & 38 & gi:15925597 & Membrane \\
\hline 35 & 505 ribosomal protein & Protein synthesis & 174 & $4.5 / 13$ & 96 & gi:15923530 & transmembrane \\
\hline 38 & 505 ribosomal protein & Protein synthesis & 210 & $9.8 / 20$ & 77 & gi:15925228 & Cytosolic \\
\hline 39 & 505 ribosomal protein & Protein synthesis & 78 & $9.9 / 20$ & 52 & gi:15925225 & Cytosolic \\
\hline 40 & $50 S$ ribosomal protein & Protein synthesis & 50 & $10.8 / 16$ & 43 & gi:15925221 & Cytosolic \\
\hline 41 & 505 ribosomal protein & Protein synthesis & 70 & $9.5 / 15$ & 42 & gi:15923527 & Cytosolic \\
\hline 42 & $50 S$ ribosomal protein & Protein synthesis & 40 & $10.3 / 11$ & 50 & gi:15924637 & Cytosolic \\
\hline $36,37,43$ & $50 S$ ribosomal protein & Protein synthesis & 10 & $9.5 / 24$ & 87 & gi:15923528 & Cytosolic \\
\hline 44 & Endopeptidase & Protein degradation & 438 & $5.4 / 91$ & 57 & gi:15923515 & Cytosolic \\
\hline 45 & 305 ribosomal protein & Protein synthesis & 100 & $10.1 / 24$ & 44 & gi:15925234 & Cytosolic \\
\hline 46 & Inositol-monophosphate DH & others & 178 & $5.5 / 53$ & 45 & gi:15923380 & transmembrane \\
\hline 47 & 505 ribosomal protein & Protein synthesis & 80 & $10.2 / 24$ & 36 & gi:15925240 & Cytosolic \\
\hline 48 & Catalase & Stress protein & 164 & $5.2 / 58$ & 40 & gi:21282950 & Cytosolic \\
\hline 49 & Pyruvate dehydrogenase E1 & Virulence mechanism & 90 & $4.8 / 41$ & 24 & gi:15924083 & Membrane \\
\hline
\end{tabular}


Table 1 Proteins shown in the reference map were identified with a linear ion trap nano-LC-ESI-MS/MS (Continued)

\begin{tabular}{|c|c|c|c|c|c|c|c|}
\hline 50 & Threonine dehydrogenase & Aminio acid metabolism & 166 & $6.4 / 37$ & 35 & gi:15924428 & Membrane \\
\hline 51 & Poly.phosphorlase/adenylase & Others & 268 & $4.7 / 77$ & 40 & gi:15924264 & transmembrane \\
\hline 52 & Glutamyl-tRNA synthetase & Cell division & 116 & $5.0 / 56$ & 31 & gi:15923518 & transmembrane \\
\hline 53 & 505 ribosomal protein & Protein Synthesis & 60 & $10.3 / 24$ & 17 & gi:15925240 & Cytosolic \\
\hline 54 & $\mathrm{ABC}$ transporter & Cell division & 100 & $5.37 / 48$ & 32 & gi:15923833 & transmembrane \\
\hline 55 & Adenylosuccinate synthetase & Others & 150 & $5.0 / 48$ & 41 & gi:15923007 & Membrane \\
\hline 56 & Alkyl hydroperoxide reductase & Stress protein & 160 & $4.6 / 55$ & 32 & gi:15923370 & transmembrane \\
\hline 57 & Akaline shock protein 23 & Stress protein & 156 & $5.0 / 20$ & 48 & gi:15925172 & Cytosolic \\
\hline 58 & Hypothetical protein SAV1845 & Others & 90 & $4.1 / 13$ & 72 & gi:15924835 & Cytosolic \\
\hline 59 & Acetate kinase & Carbohydrate metabolism & 176 & $5.6 / 44$ & 61 & gi:21283383 & transmembrane \\
\hline 60 & Alcohol dehydrogenase & Carbohydrate metabolism & 140 & $5.2 / 36$ & 48 & gi:21282297 & transmembrane \\
\hline 61 & Succinyl-CoA synthetase & Carbohydrate metabolism & 46 & $5.4 / 32$ & 16 & gi:15924236 & Not clear \\
\hline 62 & Fructose-1,6-bioP- aldolase & Carbohydrate metabolism & 190 & $4.7 / 33$ & 50 & gi:15925596 & Cytosolic \\
\hline 63 & Acid decarboxylase & Carbohydrate metabolism & 240 & $4.7 / 33$ & 78 & gi:15925596 & Cytosolic \\
\hline 64 & Fructose-bi-P-aldolase & Carbohydrate metabolism & 70 & $4.9 / 31$ & 20 & gi:15925115 & Not clear \\
\hline 65 & Phenylalanyl-tRNA synthetase & Aminio acid metabolism & 60 & $4.9 / 22$ & 37 & gi:15924732 & Cytosolic \\
\hline 66 & Glycerate dehydrogenase & Carbohydrate metabolism & 60 & $5.0 / 35$ & 30 & gi:15925295 & Membrane \\
\hline 67 & Malate-dehydrogenase & Carbohydrate metabolism & 50 & $4.9 / 44$ & 17 & gi:15924692 & Membrane \\
\hline 68 & Ornithine-acid transaminase & Aminio acid metabolism & 90 & $5.0 / 43$ & 40 & gi:15923947 & Membrane \\
\hline $30,34,69$ & Alkyl hydroperoxide reductase & Stress protein & 30 & $4.7 / 21$ & 26 & gi:15923371 & Cytosolic \\
\hline
\end{tabular}

Gels similar to those shown in Figure 1 were subjected to image analysis to identify differentially expressed proteins. These spots were then cut from the gels, and proteins were identified via tandem mass spectrometry (MS). Protein identification was performed against NCBInr (nr.gz) databases for Staphylococcus genus. Cross correlation (XC) score is a metric associated with the quality of identification, with a score $>20$ indicating a positive identification; pl/Mr is isoelectric point and molecular weight (theoretical); Cov (\%) is the fraction of protein sequence coverage identified via MS; Access. No. is accession number for the protein.

from cytoplasmic origin is not clear. One possible reason is the inherent chemistry of lysostaphin, the enzyme used in digesting the membrane fraction. A recent study reported that the treatment of bacterial cells with lysostaphin decreases cell wall stiffness by digesting peptidoglycan and could eventually lead to the formation of osmotically fragile cells [24]. A detailed explanation of how lysostaphin causes cell autolysis during digestion is available elsewhere [25]. Alternatively, there is immunological and biochemical evidence that some of the cytoplasmic proteins may be recycled between the cytosol and membrane compartments to perform their role as a cellular scavenger of degradable proteins and carrier of newly synthesized proteins [26]. Similar to this study, contaminations of cytosolic proteins to membrane fractions in bacterial protein extraction were reported elsewhere [25,27-29]. Regardless of the origins, the presence of these cytoplasmic proteins does not diminish the significance of the membrane proteins that were identified in this study. In fact, there are reports that suggest that some of the $S$. aureus cytoplasmic proteins may have a "moonlighting function" and play roles in virulence [30,31].

Proteins visualized in the 2-DE profile were also classified based on their functions using PSORT and TMpred. About $30 \%$ of the identified proteins were involved in carbohydrate metabolism, $20 \%$ in protein synthesis and $12 \%$ in stress tolerance. KEGG prediction confirmed the functional categories of three virulence mechanism associated proteins: fibronectin-binding protein (spot 1), collagen-binding surface protein (Cna; spot 4) and trigger factor (spot 5). Fibronectin-binding protein in S. aureus is central to the invasion of endothelium. During infection, $S$. aureus forms a bridge by using fibronectin-binding proteins with the host cell receptors [29]. The collagen binding protein (Cna; spot 4) identified in this study is well studied as well for its binding with collagen [32-34]. Unlike fibrinogen- and collagen-binding proteins, the associations of elongation factor $\mathrm{Tu}$ (spots 16 and17) and pyruvate dehydrogenase (spot 18) with virulence mechanism could not be predicted by KEGG. On the contrary, association of these proteins with virulence mechanism were reported from other findings [35].

Effect of fluid shear rate on protein expression in biofilms Fluid shear, a hydrodynamic force also generated by blood flow against the vessel wall, is an important physiological phenomenon in the vasculature system [36-38]. Using physiologically relevant fluid shear rate conditions, adequate number of biofilm cells could be harvested to carry 
out 2-DE for protein separation from both cytoplasmic and membrane cell fractions (Figure 2).

Fluid shear rates range from $40 \mathrm{~s}^{-1}$ to $2,000 \mathrm{~s}^{-1}$ in physiological conditions [39].To investigate the effect of fluid shear on protein profile in biofilm, the protein expression patterns of biofilm cells grown at $50 \mathrm{~s}^{-1}, 100 \mathrm{~s}^{-1}$, $500 \mathrm{~s}^{-1}$ and $1000 \mathrm{~s}^{-1}$ were compared. Of the 200 protein spots resolved in 2-DE gels from the biofilm cells grown in shake flasks, 16 protein spots showed statistically significant differences in their abundance as shear rate was increased $(p<0.05$, Student's $t$-test). These proteins were identified using nano-LC-MS/MS. Their localization and possible functions were predicted by PSORT and TMpred (Figure 3). All the proteins with a differential spot volume in membrane fraction were confirmed as either membrane or transmembrane in their sub-cellular localization. Similarly, eight cytosolic proteins that displayed lower abundance with an increase in the fluid shear rates were identified. Sub-cellular location and function of these proteins in cytoplasm were also confirmed by PSORT and TMpred.

As shown in Figure 4, three membrane proteins showed higher abundance in biofilm cells at $1000 \mathrm{~s}^{-1}$ when compared to those at $50 \mathrm{~s}^{-1}, 100 \mathrm{~s}^{-1}$, and $500 \mathrm{~s}^{-1}$. These proteins were identified by LC-MS/MS as malate dehydrogenase (spot 20), branched-chain alpha-keto acid dehydrogenase (spot 33) and $50 \mathrm{~S}$ ribosomal protein (spot 42; Figure 4 and Table 2). Unlike the membrane associated fraction, cytoplasmic proteins that showed higher abundance with an increase in the fluid shears could not be detected.

Proteins that showed decreased expression with increasing fluid shear rates were also observed. Thirteen

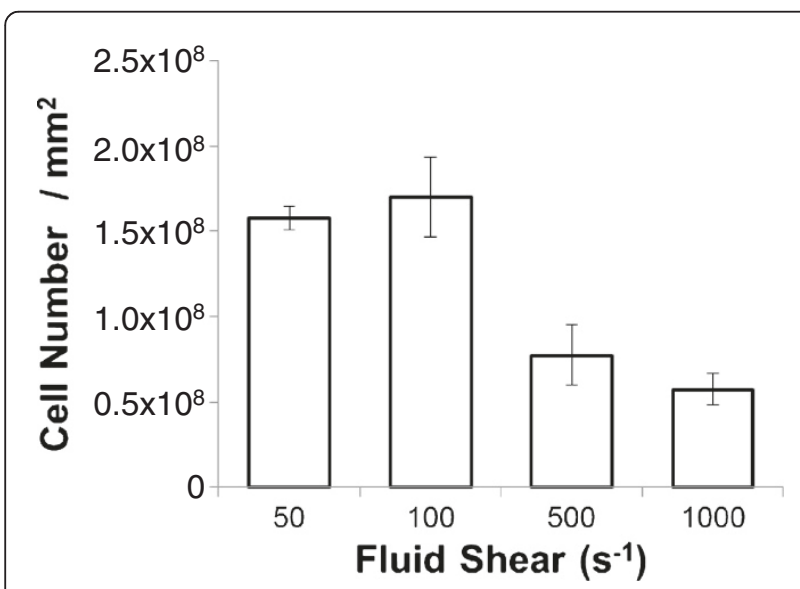

Figure 2 Number of biofilm cells collected from flow chamber system at each of four different shear rates (Average \pm standard deviations). Four biological replicates were carried out at each shear rate. As described in Methods section, biofilm cells were grown for 24 hrs with continuous supply of sterile tryptic soybroth. membrane associated proteins showed lower expression in biofilm cells at $1000 \mathrm{~s}^{-1}$ when compared to those at $50 \mathrm{~s}^{-1}, 100 \mathrm{~s}^{-1}$ and $500 \mathrm{~s}^{-1}$ (Figure 4 and Table 2). A closer look at the functional categories of proteins revealed that this decrease in protein expression represented a regulated state of lower metabolic activity. Nine of the 13 identified proteins are associated mainly in glycolysis/TCA cycle, indicating that utility of the glycolysis/TCA pathways were minimized under high shear rates. Glyceraldehyde 3-phosphate dehydrogenase decreased (spot 2 and 3) by 4.2 to 5.8 folds in $1000 \mathrm{~s}^{-1}$ when compared to that in $50 \mathrm{~s}^{-1}$.

Similar to phosphate dehydrogenase, alanine dehydrogenase (spot 4), another important protein that catalyzes the NAD-dependent reversible reductive amination of pyruvate into alanine, was decreased by 4.2 fold in $1000 \mathrm{~s}^{-1}$ when compared to $50 \mathrm{~s}^{-1}$ (Table 2 and Figure 4). In addition, a significant decrease in malate:quinone oxidoreductase and succinyl-CoA synthase were observed under high fluid shear rates.

In cytosolic fraction, eight proteins showed lower abundance at $1000 \mathrm{~s}^{-1}$ when compared to $50 \mathrm{~s}^{-1}$. Like with the membrane protein fraction, glyceraldehyde-3-phosphate dehydrogenase decreased significantly at $1000 \mathrm{~s}^{-1}$ when compared to $50 \mathrm{~s}^{-1}$. In addition, a lower abundance of alcohol dehydrogenase, succinyl-CoA synthetase, formate acetyltransferase, and malate dehydrogenase was observed under high fluid shear stress.

In the proteome profile reported here, six virulenceassociated surface proteins were identified: fibronectinbinding protein (spot 1), collagen-binding surface protein (Cna; spot 4), trigger factor (spot 5), elongation factor Tu (spots 16 and17) and pyruvate dehydrogenase (spot 18). However, changes in expression of these proteins were not detectable under fluid shear stress. On the contrary, most of the glycolysis / TCA cycle associated proteins decreased significantly under higher shear rates. The data thus demonstrate for the first time the relationship between metabolic activity and virulence potential. The glycolysis/TCA metabolic activity of $S$. aureus was low under fluid high shear, while the abundance of surface proteins, especially the fibronectin-binding and collagen binding surface proteins, remained unchanged. Although the ability of these cells in neo-colonization was not specifically tested, the unchanged abundance of these surface binding proteins implies that these cells retain their ability for further infection. Alternatively, there might be an interaction between virulence mechanism associated proteins and glycolysis/ TCA associated proteins under high fluid shear that is yet to be determined. As glucose is the main metabolite of the glycolysis/TCA pathways, it is anticipated that carbohydrate metabolism might have potential contribution to the virulence mechanism associated proteins. Such an interaction of carbohydrate structures and their associated 

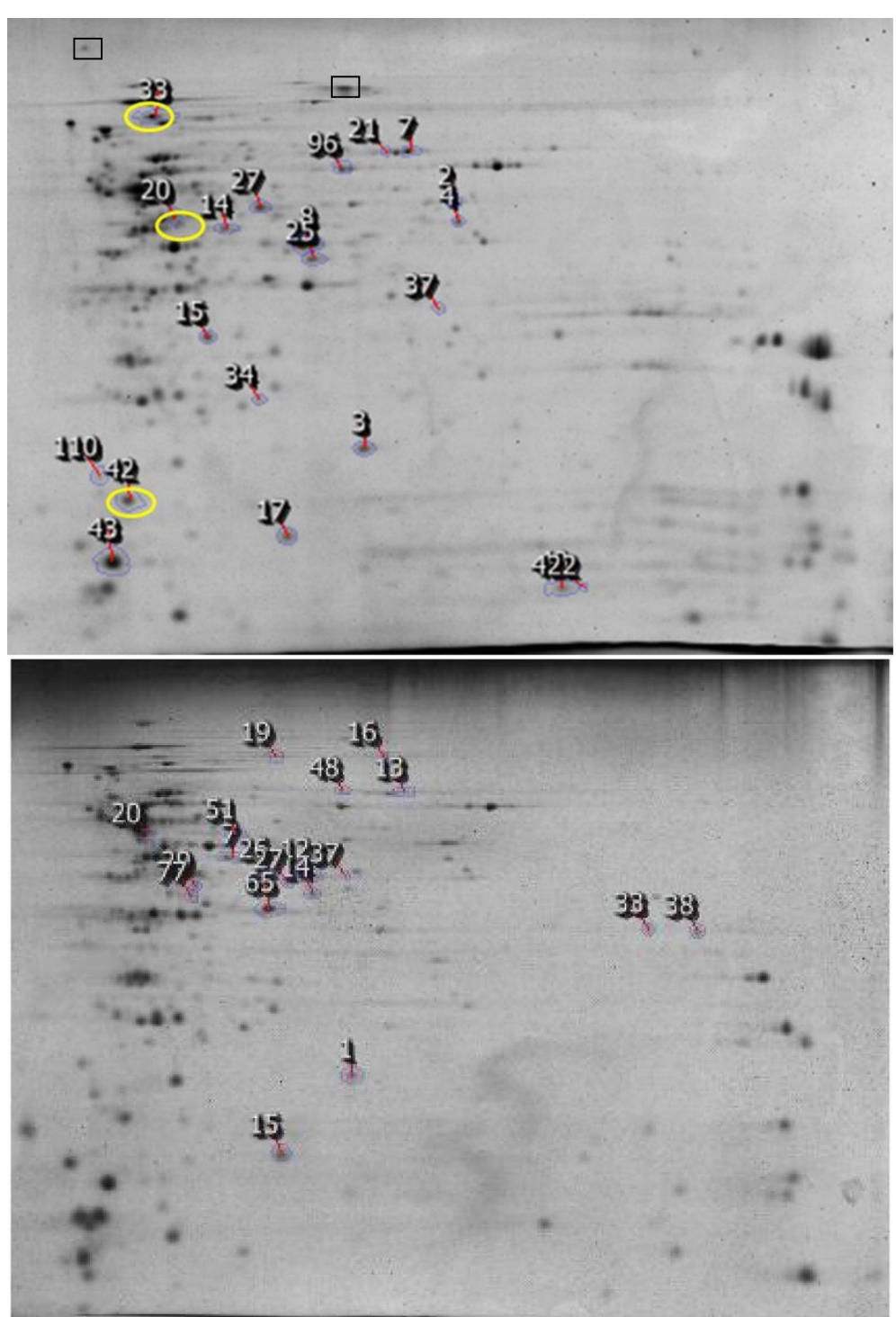

Figure 3 2-DE gel images of membrane associated fraction (top) and cytosolic fraction (bottom) of S. aureus biofilm cells grown at fluid shear rate of $1000 \mathrm{~s}^{-1}$. Circled spot ID numbers correspond to the proteins with increased level of expression at $1000 \mathrm{~s}^{-1}$. Differentially expressed protein were identified and shown in Table 2. Corresponding position of fibronectin-binding protein (spot 1 in Figure 1) and collagen-binding protein (Cna; spot 4 in Figure 1) are shown in rectangular box.

proteins with cell wall-anchored proteins was predicted from other findings [25].

\section{Metabolic pathways of identified proteins}

Some of the identified proteins were mapped in their metabolic pathways (Figure 5). As observed in Table 2, most of the changed proteins were associated with carbohydrate metabolism especially glycolysis and TCA cycle. Glyceraldehyde 3-phosphate dehydrogenase (GapC; spots M2 and M3, C1) reduced significantly as fluid shear rates were increased. This protein is responsible for the inter-conversion of 1,3-diphosphoglycerate and glyceraldehyde-3-phosphate (GAPDH), a central step in glycolysis which utilizes NAD for phosphorylation. As fluid shear significantly decreased the abundance of this protein, less energy is anticipated to be available for the transfer of inorganic phosphate to high phosphoryl transfer product, indicating that the bacteria exhibits lower metabolic activity under higher fluid shear stress. Although cytosol is considered to be a primary location for this protein, it was recently reported that GAPDH and several other glycolytic enzymes assemble in complexes on the inside of the cell membrane [26]. This protein is also considered as a metabolic switch for rerouting the carbohydrate flux to counteract stress [40]. 


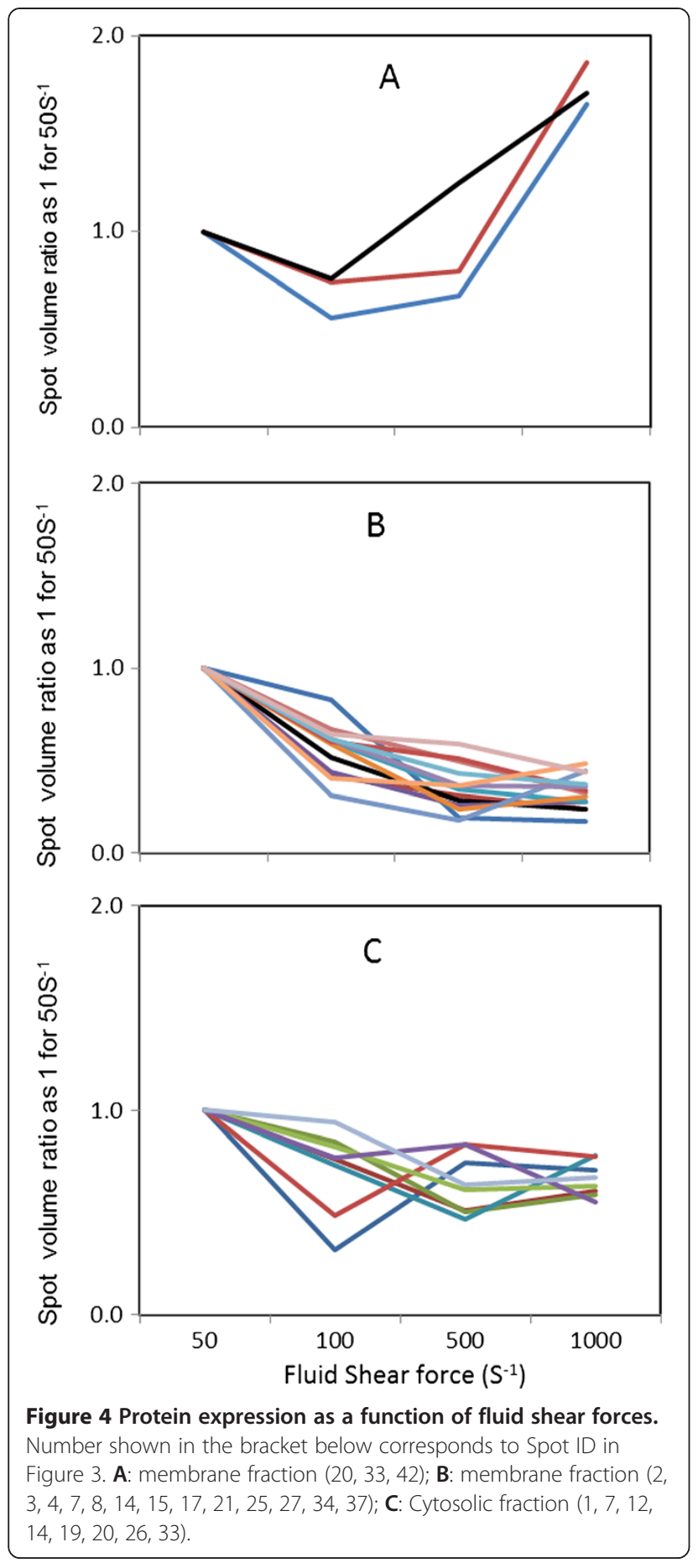

In addition to a reduction in GAPDH under high fluid shears, significant decreases in alanine dehydrogenase and formate acetyltransferase were also observed. Both of these enzymes provide pyruvate for glycolytic pathways. Reduction in these enzymes also implies lower acetyl-CoA that drives TCA cycles.

There were significant changes in three enzymes of TCA cycle with the increased rate of fluid shear (Figure 5).
Malate dehydrogenase that catalyzes the conversion of malate into oxaloacetate (using NAD+) increased significantly. This increase in malate dehydrogenase seems to contradict with the decreased level of expression of other glycolytic and TCA cycle enzymes in this study. However, the reaction mediated by this enzyme is reversible, indicating that the increased level in malate dehydrogenase might have resulted due to the reverse reaction. This is also consistent with the reduction in malate:quinone oxidoreductase (spot 7). In addition, a significant decrease in the expression of succnyl-CoA synthetase was also observed. Taken together, it can be concluded that fluid shear stress have a significant effect on the abundance of some key enzymes in the TCA cycles, implying lower TCA cycle activity.

Implications of reduced TCA cycle activity in regulating or affecting staphylococcal virulence and/or virulence determinant biosynthesis are well studied [41-45]. Higher amount of capsular polysaccharides in relation to TCA cycle inactivation was reported [41]. It was anticipated that TCA cycle inactivation results lower amount of oxaloacetate for conversion to phosphoenoylpyruvate to be used in gluconeogensis pathway [41].

Inhibiting TCA cycle activity dramatically increases polysaccharides intercellular adhesins (PIA) biosynthesis through genetic regulation of icaADBC, a group of genes responsible for production of PIA [43,45]. It is anticipated that regulatory proteins responding to TCA cycle-mediated changes in the metabolic status regulate ica $A D B C$ transcription, indicating that the TCA cycle acts as a signal transduction pathways for regulating virulence factor synthesis and biofilm formation [41]. Lower glycolytic and TCA cycle activities of bacteria under fluid shear stress is also consistent with lower amount of cell that was harvested at 500 and $1000 \mathrm{~s}^{-1}$ (Figure 2).

Here, it is demonstrated that under fluid shear conditions, bacteria maintain a dormant mode of growth by reducing glycolytic and TCA cycles activities for their survival while maintaining their capacity for further infection. From clinical perspective, the dormant mode of growth is considered a major role that bacteria plays to escape the effect of antibiotics [46].

\section{Conclusions}

The expression levels of two important surface binding proteins, fibronectin-binding and collagen-binding proteins, were not influenced by varying levels of physiological fluid shear rates. On the other hand, some of the $S$. aureus proteins that are associated with metabolic functions such as carbohydrates, protein synthesis, and stress tolerance significantly changed their level of expression. These results imply that bacteria maintain slow metabolic activity under high fluid shear stress. To 
Table 2 Differentially expressed membrane and cytosolic protein fractions were identified with linear ion trap nano-LC-ESI-MS/MS

\begin{tabular}{|c|c|c|c|c|c|c|c|}
\hline Spot ID & Fold changed & Protein name & XC score & $\operatorname{Cov}(\%)$ & $\mathrm{pl} / \mathrm{Mr}$ & Acc no & Funtional category \\
\hline \multicolumn{8}{|c|}{ Membrane fraction: higher abundance in 1000S-1 } \\
\hline 20 & -3.1 & Malate dehydrogenase & 170 & 54 & $4.9 / 44$ & gi:15924692 & Carbohydrate metabolism \\
\hline 33 & -2.5 & Brached -chain alpha-keto acid dehydrogenase & 260 & 42 & $4.8 / 46$ & gi:15924085 & Carbohydrate metabolism \\
\hline 42 & -2.2 & $50 S$ ribosomal protein & 80 & 58 & $4.6 / 17$ & gi:15923529 & Amino acid metabolism \\
\hline \multicolumn{8}{|c|}{ Membrane fraction: lower abundance in $1000 \mathrm{~S}-1$} \\
\hline 2 & 5.8 & Glyceraldehyde 3-phosphate dehydrogenase & 106 & 30 & $5.9 / 37$ & gi:15924677 & Carbohydrate metabolism \\
\hline 3 & 4.2 & Glyceraldehyde 3-phosphate dehydrogenase & 48 & 21 & $5.9 / 37$ & gi:15924677 & Carbohydrate metabolism \\
\hline 4 & 4.2 & Alanine dehydrogenase & 258 & 66 & $5.5 / 40$ & gi:21283381 & Carbohydrate metabolism \\
\hline 7 & 3.6 & Malate:quinone oxidoreductase & 443 & 62 & $6.1 / 56$ & gi:15925597 & Carbohydrate metabolism \\
\hline 8 & 3.6 & Alcohol dehydrogenase & 510 & 76 & $5.2 / 36$ & gi:21282297 & Carbohydrate metabolism \\
\hline 14 & 3.3 & Ornithine-oxo-acid transaminase & 340 & 81 & $5.1 / 43$ & gi:15923947 & Amino acid metabolism \\
\hline 15 & 3.2 & Ribosomal subunit interface protein & 200 & 68 & $5.0 / 22$ & gi:15923742 & Amino acid metabolism \\
\hline 17 & 3.1 & Nucleoside diphosphate kinase & 90 & 61 & $4.9 / 17$ & gi:15924459 & Nucleotide Metabolism \\
\hline 21 & 2.9 & Malate:quinone oxidoreductase & 236 & 44 & $6.1 / 56$ & gi:15925597 & Carbohydrate metabolism \\
\hline 25 & 2.8 & Succinyl-CoA synthease & 166 & 49 & $5.4 / 32$ & gi:15924236 & Carbohydrate metabolism \\
\hline 27 & 2.7 & Alanine dehydrogenase & 148 & 47 & $5.0 / 40$ & gi:15924429 & Carbohydrate metabolism \\
\hline 34 & 2.5 & Superoxide dismutase & 118 & 62 & $5.2 / 23$ & gi:15923123 & Stress protein \\
\hline 37 & 2.4 & FOF1 ATP synthase & 80 & 43 & $5.7 / 32$ & gi:15925094 & Carbohydrate metabolism \\
\hline \multicolumn{8}{|c|}{ Cytosolic fraction: lower abundance in 1000S-1 } \\
\hline 1 & 0.71 & Glyceraldehyde 3-phosphate dehydrogenase & 48 & 21 & $5.9 / 37$ & gi:15924677 & Carbohydrate metabolism \\
\hline 7 & 0.60 & Ornithine-oxo-acid transaminase & 340 & 81 & $5.1 / 43$ & gi:15923947 & Amino acid metabolism \\
\hline 12 & 0.59 & Alcohol dehydrogenase & 516 & 76 & $5.2 / 36$ & gi:21282297 & Carbohydrate metabolism \\
\hline 14 & 0.78 & Succinyl-CoA synthetase & 106 & 30 & $5.4 / 32$ & gi:15924236 & Carbohydrate metabolism \\
\hline 19 & 0.77 & Formate acetyltransferase & 148 & 22 & $5.2 / 84$ & gi:15923216 & Carbohydrate metabolism \\
\hline 20 & 0.63 & Malate dehydrogenase & 170 & 54 & $4.9 / 44$ & gi:15924692 & Carbohydrate metabolism \\
\hline 26 & 0.55 & Alcohol dehydrogenase & 510 & 76 & $5.2 / 36$ & gi:21282297 & Carbohydrate metabolism \\
\hline 33 & 0.67 & $50 \mathrm{~S}$ ribosomal protein & 80 & 36 & $10.2 / 24$ & gi:15925240 & Amino acid metabolism \\
\hline
\end{tabular}

Fold change was calculated using proteins in $50 \mathrm{~s}^{-1}$ compared to those in $1000 \mathrm{~s}^{-1}$. Protein functional categories were annotated from KEGG database.

our knowledge, this is the first report that catalogs the differential protein expression under various physiologically relevant fluid shear stress conditions. As revealed from this study, most of the altered proteins were directly related to the energy balance of different metabolic pathways. A future study that elucidates post-translational protein modifications (especially phosphorylation) may provide a further understanding of the highly variable biofilm proteomes under fluid shear forces.

\section{Methods}

Bacterial culture

S. aureus Phillips, a biofilm forming bacteria, was used in this study. The strain was isolated from a patient diagnosed with osteomyelitis and has been extensively studied $[9,10,33]$. S. aureus cultures were started by inoculation $(10 \mu \mathrm{L})$ from glycerol stocks into tryptic soy broth (TSB;
$50 \mu \mathrm{L})$ supplemented with $0.25 \%(\mathrm{w} / \mathrm{v})$ glucose and grown at $37^{\circ} \mathrm{C}$ with constant rotation at $141 \mathrm{rpm}$ in shake flasks. The growth of the bacterial strains was monitored by measuring the absorbance of the broth at $600 \mathrm{~nm}$ on a spectrophotometer. The cells were harvested at mid-exponential phase $\left(\mathrm{OD}_{600}=0.3\right.$ to 0.35$)$, centrifuged $(4000 \mathrm{rpm})$ for $15 \mathrm{~min}$ at $4^{\circ} \mathrm{C}$ and resuspended in phosphate-buffered saline (DPBS; $138 \mathrm{mM} \mathrm{NaCl}, 2.7 \mathrm{mM}$ $\mathrm{KCl}, \mathrm{pH}$ 7.4). Cell concentrations were determined using a Coulter Multisizer (Beckman Coulter).

\section{Flow chamber system}

A $40 \mathrm{~cm}$ silicon tube (SILASTIC) was used as the reactor. Briefly, after sterilization, the reactor tube was filled with collagen type I for coating and washed with sterile PBS after an hour. The reactor was then inoculated with 20-fold diluted mid-exponential phase cells 


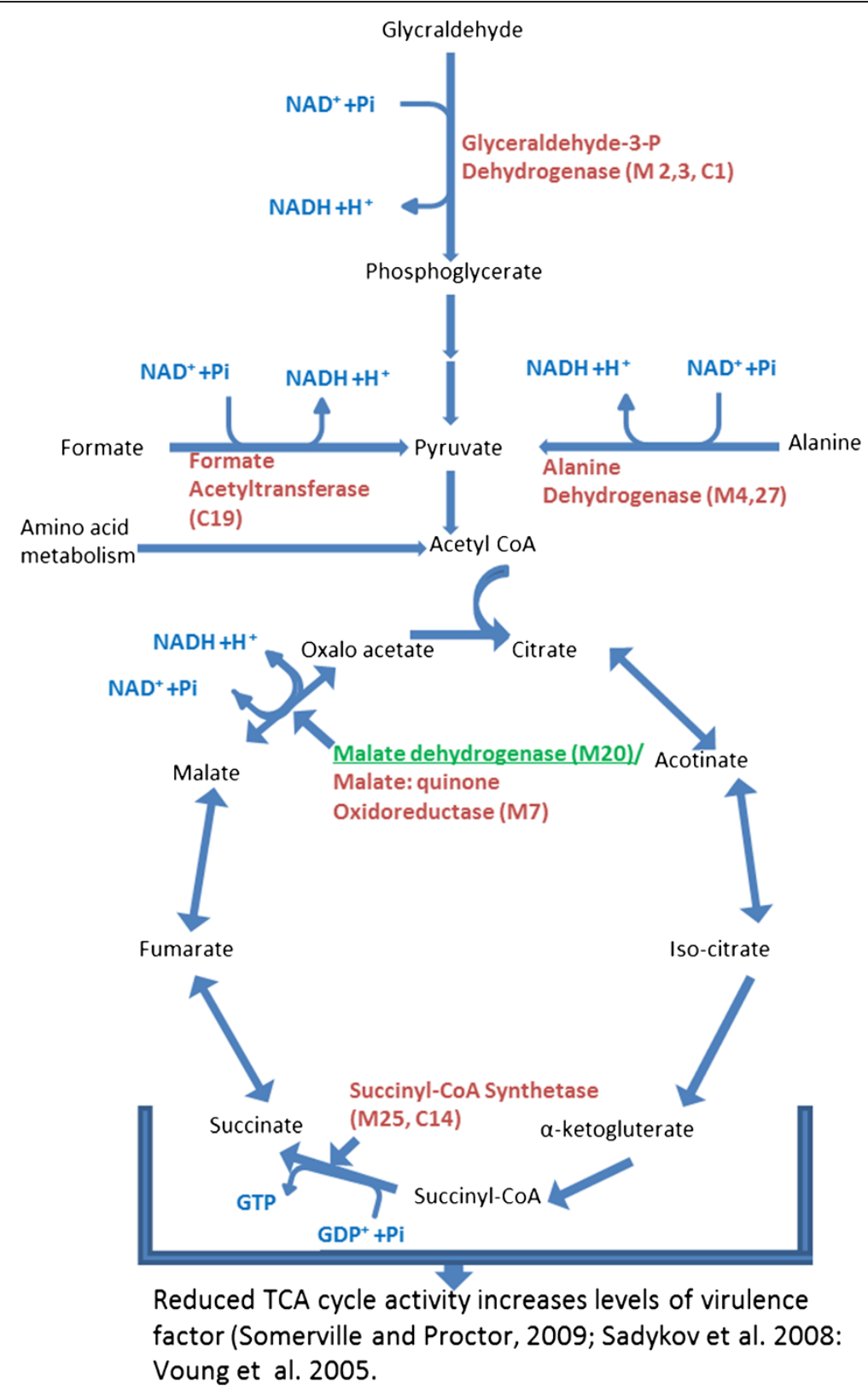

Figure 5 Metabolic pathways (glycolysis and TCA cycle) of differentially expressed proteins. Bolded names denote proteins with decreased abundance at $1000 \mathrm{~s}^{-1}$ when compared to $50 \mathrm{~s}^{-1}, 100 \mathrm{~s}^{-1}$ and $500 \mathrm{~s}^{-1}$, while underlined names denote proteins with increased expression. Lower abundance of key enzymes in the TCA cycles increased levels of virulent factors $[41,43,45]$.

for 10 minutes. The unattached cells were then rinsed out of the silicon tubing with sterile PBS buffer. One end of the reactor was then connected to a continuous supply of TSB using a peristaltic pump which was calibrated for different flow rates to provide the desired shear stresses. The wall shear rate $(\dot{\gamma})$ in inverse seconds, assuming Newtonian fluid behavior and constant density and viscosity, was calculated by the formula: $\dot{\gamma}=$ $32 \mathrm{Q} / \pi \mathrm{D}^{3}$, where $\mathrm{Q}$ is the volumetric flow rate and $\mathrm{D}$ is the tube internal diameter. The equation and further explanation of flow rates and shear stress calculations can be found in a recent review [47]. During all experiments, the entire flow system was maintained at $37^{\circ} \mathrm{C}$. After 24 hours, the reactor tube was gently removed from the system. Biofilm cells were extruded by squeezing the reactor tube. The tube was washed three times with phosphate-buffered saline to ensure recovery of all biofilm cells from the tube. After brief sonication, the cell concentration was measured using the Coulter Multisizer. 


\section{Protein extraction}

Cells were washed with PBS containing 0.1\% sodium azide and then with PBS without azide followed by a brief wash with digestion buffer containing $10 \mathrm{mM}$ Tris $\mathrm{HCl}, 1 \mathrm{mM}$ EDTA, $5 \mathrm{mM} \mathrm{MgCl} 2$. Approximately $5 \times 10^{9}$ bacterial cells were resuspended in $1 \mathrm{~mL}$ of digestion mixture containing 35\% raffinose (Sigma), protease inhibitor cocktail (Roche; 1 tablet/mL of digestion buffer), and lysostaphin (Sigma; 5 units $/ \mathrm{mL}$ ). The cells were incubated in this lysis buffer at $37^{\circ} \mathrm{C}$ for $30 \mathrm{~min}$. Cell debris was removed by centrifugation at $8,000 \mathrm{~g}$ for 20 minutes. The digest was kept at $-20^{\circ} \mathrm{C}$ overnight and then centrifuged at 8,000 $g$ for $20 \mathrm{~min}$; precipitated raffinose was discarded. The protein solution was subjected to ultrafiltration (Millipore) per manufacturer's instructions. Membrane proteins were extracted in 2-DE solubilization buffer urea ( $8 \mathrm{M}$; Sigma), thiourea (2 M; Sigma), ASB 14 (1\%; Sigma) and DTT (1\%; Sigma), carrier ampholytes (0.08\%; GE Healthcare)] with sonication. Protein concentration in the solution was determined using 2D Quant (GE Healthcare), and the resulting solution was stored at $-80^{\circ} \mathrm{C}$ for $2-\mathrm{DE}$.

\section{Two dimensional Gel electrophoresis}

In preparation for 2-DE, $150 \mu \mathrm{g}$ proteins were resolubilized by adding 2-DE solubilization buffer. The mixture was vortexed intermittently until the protein was completely solubilized. The resulting solution was then diluted to the desired volume with destreak rehydration solutions (GE Healthcare). Rehydration of IPG strips (pH 3-11, NL 24 cm; GE Healthcare) with the sample was carried out in the Immobiline Dry Strip Re-swelling Tray (GE Healthcare) according to the manufacturer's instructions. The rehydrated strips were then subjected to IEF using IPGphor (GE Healthcare) operated at $20^{\circ} \mathrm{C}$ in gradient mode $(97 \mathrm{kVhr})$. After focusing, the strips were stored at $-80^{\circ} \mathrm{C}$ for later use. Prior to the second dimension SDS-PAGE, IPG strips were equilibrated for 15 minutes in equilibration solution $(15 \mathrm{~mL})$ containing $50 \mathrm{mM}$ Tris- $\mathrm{HCl}, \mathrm{pH} 8.8,6 \mathrm{M}$ urea, 30\% w/v glycerol, $2 \% \mathrm{w} / \mathrm{v}$ SDS and traces of bromophenol blue with $10 \mathrm{mg} / \mathrm{mL}(\mathrm{w} / \mathrm{v})$ of DTT. A second equilibration was carried out for 15 minutes by adding iodoacetamide $(25 \mathrm{mg} / \mathrm{mL})$ instead of DTT in equilibration solution. Second dimension SDS-PAGE was performed using large format $(26.8 \times 20.5 \mathrm{~cm})$ gels $(12.5 \% \mathrm{~T} / 2.6 \% \mathrm{C})$ according to the manufacturer's instructions. Electrophoresis was carried out with an initial constant voltage of $10 \mathrm{~mA} /$ gel applied for 30 minutes followed by $20 \mathrm{~mA} / \mathrm{gel}$ for overnight until the bromophenol band exited the gel. The gels were stained with Colloidal Coomassie brilliant blue (BioRad). Briefly, gels were washed two times using distilled water and then placed on Dodeca high throughput gel staining tray (BioRad) followed by destaining using distilled water. All steps were carried out at room temperature. Gels were scanned as 12-bit TIFF images using GS-800 densitometer (BioRad) and analyzed by Nonlinear Dynamics SameSpots (v.3.2). Spot volumes were normalized by the software to a reference gel. At least three gels (biological replicates) for each treatment were used for analyses. Student's $t$-test was performed, and spots with more than 2-fold were considered as significant.

\section{Protein identification}

For mass spectrometric identification, gel spots were excised, destained, and digested with sequencing grade trypsin (Promega) as described elsewhere [48]. Peptide samples were then analyzed by nano-LC-ESI-MS/MS using LTQ (Finnigan, Thermo, USA). Nano-LC was performed at reversed phase conditions using Ultimate 3000 (Dionex corporation, USA) C18 column with a flow rate of $1-5 \mu \mathrm{L} / \mathrm{min}$ in $70-90 \%$ acetonitrile containing $0.1 \%$ formic acid. MS and MS/MS data were collected and interrogated against the NCBI non-redundant protein database for $S$. aureus using SEQUEST with a peptide tolerance of $1.4 \mathrm{amu}$. Searched results were then filtered using three criteria: distinct peptides, $\mathrm{XC}$ score vs Charge state $(1.50,2.00,2.50,3.00)$ and peptide probability (0.001). The confirmation of the protein identification was based on the XC score value of more than 50 and Sf score for individual peptide of more than 0.8 . It is to be noted that more than one protein were found in each spot, which is not unusual for a gel-based protein separation. Therefore, protein with the highest XC for each spot was selected as positive identification.

\section{Protein localization and function}

The prediction of protein localization sites in cells was determined by PSORT, a computer program which analyzes the input sequence by applying the stored rules for various sequence features of known protein sorting signals. The transmembrane protein domain was predicted by TMpred (http://www.ch.embnet.org/software/TMPRED_form.html). To analyze functional categories of the identified proteins, they were submitted to the KEGG database (http://www.genome.ad.jp/kegg/pathway.html) using BRITE hierarchy. KEGG BRITE is a collection of hierarchical classifications of proteins based on their biological function.

\section{Competing interests}

The authors declare that there are no competing interests.

\section{Authors' contributions}

$\mathrm{NI}$ designed and conducted the experiment and is the corresponding author of this manuscript. JMR and MRM developed the concept. NI and YK analyzed the data and wrote the manuscript. All authors read and approved the final manuscript. 


\section{Acknowledgment}

This research was supported by Grant R01-Al059369 from the National Institutes of Health (NIH), USA, and by Grant EEC-1342388 from the National Science Foundation (NSF), USA. We thank Drs. Bin Guan and Alexei Gapeev for their help with nano-LC-ESI-MS/MS, and special thanks to Dr. Pyong Kyun Shin for helping $\mathrm{NI}$ in setting up the biofilm experiment.

\section{Author details}

${ }^{1}$ Department of Chemical, Biochemical and Environmental Engineering, University of Maryland Baltimore County (UMBC), Baltimore, MD 21250, USA. ${ }^{2}$ Department of Chemical and Biological Engineering, The University of Alabama, Tuscaloosa, AL 35487, USA. ${ }^{3}$ Current address: Department of Plant Sciences, University of Maryland, College Park, MD 20742, USA.

Received: 5 December 2013 Accepted: 17 April 2014 Published: 30 April 2014

\section{References}

1. Chen J, Fallarero A, Määttänen A, Sandberg M, Peltonen J, Vuorela P, Riekkola M: Living cells of Staphylococcus aureus immobilized onto the capillary surface in electrochromatography: a tool for screening of biofilms. Anal Chem 2008, 80:5103-5109.

2. Mah T, O'Toole G: Mechanisms of biofilm resistance to antimicrobial agents. Trends Microbiol 2001, 9:34-39.

3. Stewart $P$, Costerton J: Antibiotic resistance of bacteria in biofilms. Lancet 2001, 358:135-138

4. Donlan R, Costerton J: Biofilms: survival mechanisms of clinically relevant microorganisms. Clin Microbiol Rev 2002, 15:167-193.

5. Macia MD, Perez JL, Molin S, Oliver A: Dynamics of mutator and antibioticresistant populations in a pharmacokinetic/pharmacodynamic model of Pseudomonas aeruginosa biofilm treatment. Antimicrob Agents Chemother 2011, 55:5230-5237.

6. Stoodley P, Lewandowski Z, Boyle J, Lappin-Scott H: Oscillation characteristics of biofilm streamers in turbulent flowing water as related to drag and pressure drop. Biotechnol Bioeng 1998, 57:536-544.

7. Mohamed N, Teeters MA, Patti JM, Hook M, Ross JM: Inhibition of Staphylococcus aureus adherence to collagen under dynamic conditions. Infect Immun 1999, 67:589-594.

8. Mohamed N, Rainier TR Jr, Ross JM: Novel experimental study of receptormediated bacterial adhesion under the influence of fluid shear. Biotechnol Bioeng 2000, 68:628-636.

9. George NP, Wei Q, Shin PK, Konstantopoulos K, Ross JM: Staphylococcus aureus adhesion via Spa, CIfA, and SdrCDE to immobilized platelets demonstrates shear-dependent behavior. Arterioscler Thromb Vasc Biol 2006, 26:2394-2400.

10. George NP, Konstantopoulos K, Ross JM: Differential kinetics and molecular recognition mechanisms involved in early versus late growth phase Staphylococcus aureus cell binding to platelet layers under physiological shear conditions. J Infect Dis 2007, 196:639-646.

11. Johnson MA, Ross JM: Staphylococcal presence alters thrombus formation under physiological shear conditions in whole blood studies. Ann Biomed Eng 2008, 36:349-355.

12. Reddy K, Ross JM: Shear stress prevents fibronectin binding proteinmediated Staphylococcus aureus adhesion to resting endothelial cells. Infect Immun 2001, 69:3472-3475.

13. Ymele-Leki P, Ross JM: Erosion from Staphylococcus aureus biofilms grown under physiologically relevant fluid shear forces yields bacterial cells with reduced avidity to collagen. Appl Environ Microbiol 2007, 73:1834-1841.

14. Engelmann S, Hecker M: Proteomic analysis to investigate regulatory networks in Staphylococcus aureus. Methods Mol Biol 2008, 431:25-45.

15. Kohler C, Wolff S, Albrecht D, Fuchs S, Becher D, Büttner K, Engelmann S, Hecker M: Proteome analyses of Staphylococcus aureus in growing and non-growing cells: a physiological approach. Int J Med Microbio/ 2005, 295:547-565.

16. Wolf $C$, Hochgräfe F, Kusch H, Albrecht D, Hecker M, Engelmann S: Proteomic analysis of antioxidant strategies of Staphylococcus aureus: diverse responses to different oxidants. Proteomics 2008, 8:3139-3153.

17. Resch A, Leicht S, Saric M, Pásztor L, Jakob A, Götz F, Nordheim A: Comparative proteome analysis of Staphylococcus aureus biofilm and planktonic cells and correlation with transcriptome profiling. Proteomics 2006, 6:1867-1877.

18. Cohen P: Community-acquired methicillin-resistant Staphylococcus aureus skin infections: implications for patients and practitioners. Am J Clin Dermatol 2007, 8:259-270.

19. Prigent-Combaret $C$, Lejeune P: Monitoring gene expression in biofilms. Methods Enzymol 1999, 310:56-79.

20. Clements M, Watson S, Foster S: Characterization of the major superoxide dismutase of Staphylococcus aureus and its role in starvation survival, stress resistance, and pathogenicity. J Bacteriol 1999, 181:3898-3903.

21. Novick R: Autoinduction and signal transduction in the regulation of staphylococcal virulence. Mol Microbiol 2003, 48:1429-1449.

22. Nakai K, Horton P: PSORT: a program for detecting sorting signals in proteins and predicting their subcellular localization. Trends Biochem Sci 1999, 24:34-36.

23. Hofman K, Stoffel W: TMbase-A database of membrane spanning protein segments. Biol Chem Hoppe Seyler 1993, 374:166.

24. Francius G, Domenech O, Mingeot-Leclercq M, Dufrêne Y: Direct observation of Staphylococcus aureus cell wall digestion by lysostaphin. J Bacteriol 2008, 190:7904-7909.

25. Gatlin C, Pieper R, Huang S, Mongodin E, Gebregeorgis E, Parmar P, Clark D, Alami H, Papazisi L, Fleischmann R, Peterson S: Proteomic profiling of cell envelope-associated proteins from Staphylococcus aureus. Proteomics 2006, 6:1530-1549

26. Campanella M, Chu H, Low P: Assembly and regulation of a glycolytic enzyme complex on the human erythrocyte membrane. Proc Natl Acad Sci U S A 2005, 102:2402-2407.

27. Nandakumar R, Nandakumar M, Marten M, Ross J: Proteome analysis of membrane and cell wall associated proteins from Staphylococcus aureus. J Proteome Res 2005, 4:250-257.

28. Brady R, Leid J, Camper A, Costerton J, Shirtliff M: Identification of Staphylococcus aureus proteins recognized by the antibody-mediated immune response to a biofilm infection. Infect Immun 2006, 74:3415-3426.

29. Ahmed S, Meghii S, Williams R, Henderson B, Brock J, Nair S Staphylococcus aureus fibronectin binding proteins are essential for internalization by osteoblasts but do not account for differences in intracellular levels of bacteria. Infect Immun 2001, 69:2872-2877.

30. Henderson B, Martin A: Bacterial virulence in the moonlight: multitasking bacterial moonlighting proteins are virulence determinants in infectious disease. Infect Immun 2011, 79:3476-3491.

31. Henderson B, Martin A: Bacterial moonlighting proteins and bacterial virulence. Curr Top Microbiol Immunol 2013, 358:155-213.

32. Patti J, Boles J, Höök M: Identification and biochemical characterization of the ligand binding domain of the collagen adhesin from Staphylococcus aureus. Biochemistry 1993, 32:11428-11435.

33. Patti J, Bremell T, Krajewska-Pietrasik D, Abdelnour A, Tarkowski A, Rydén C, Höök M: The Staphylococcus aureus collagen adhesin is a virulence determinant in experimental septic arthritis. Infect Immun 1994, 62:152-161.

34. Li Z, Mohamed N, Ross J: Shear stress affects the kinetics of Staphylococcus aureus adhesion to collagen. Biotechnol Prog 2000, 16:1086-1090.

35. Dallo S, Kannan T, Blaylock M, Baseman J: Elongation factor Tu and E1 beta subunit of pyruvate dehydrogenase complex act as fibronectin binding proteins in Mycoplasma pneumoniae. Mol Microbiol 2002, 46:1041-1051.

36. Gonzales R, Wick T: Hemodynamic modulation of monocytic cell adherence to vascular endothelium. Ann Biomed Eng 1996, 24:382-393.

37. Jones D, Abbassi O, Mclntire L, McEver R, Smith C: P-selectin mediates neutrophil rolling on histamine-stimulated endothelial cells. Biophys $J$ 1993, 65:1560-1569.

38. Lawrence M, McIntire L, Eskin S: Effect of flow on polymorphonuclear leukocyte/endothelial cell adhesion. Blood 1987, 70:1284-1290.

39. Papaioannou TG, Stefanadis C: Vascular wall shear stress: basic principles and methods. Hellenic J Cardiol 2005, 46:9-15.

40. Ralser M, Wamelink M, Kowald A, Gerisch B, Heeren G, Struys E, Klipp E, Jakobs C, Breitenbach $M$, Lehrach $H$, Krobitsch S: Dynamic rerouting of the carbohydrate flux is key to counteracting oxidative stress. J Biol 2007, 6:10.

41. Somerville G, Proctor R: At the crossroads of bacterial metabolism and virulence factor synthesis in Staphylococci. Microbiol Mol Biol Rev 2009, 73:233-248. 
42. Bae T, Banger A, Wallace A, Glass E, Aslund F, Schneewind O, Missiakas D: Staphylococcus aureus virulence genes identified by bursa aurealis mutagenesis and nematode killing. Proc Natl Acad Sci U S A 2004, 101:12312-12317.

43. Sadykov M, Olson M, Halouska S, Zhu Y, Fey P, Powers R, Somerville G: Tricarboxylic acid cycle-dependent regulation of Staphylococcus epidermidis polysaccharide intercellular adhesin synthesis. J Bacteriol 2008, 190:7621-7632.

44. Somerville G, Cockayne A, Dürr M, Peschel A, Otto M, Musser J: Synthesis and deformylation of Staphylococcus aureus delta-toxin are linked to tricarboxylic acid cycle activity. J Bacteriol 2003, 185:6686-6694.

45. Vuong C, Kidder J, Jacobson E, Otto M, Proctor R, Somerville G: Staphylococcus epidermidis polysaccharide intercellular adhesin production significantly increases during tricarboxylic acid cycle stress. J Bacteriol 2005, 187:2967-2973.

46. Ma L, Conover M, Lu H, Parsek M, Bayles K, Wozniak D: Assembly and development of the Pseudomonas aeruginosa biofilm matrix. PLoS Pathog 2009, 5:e1000354.

47. Busscher $\mathrm{H}$, van der Mei H: Microbial adhesion in flow displacement systems. Clin Microbiol Rev 2006, 19:127-141.

48. Gharahdaghi F, Weinberg C, Meagher D, Imai B, Mische S: Mass spectrometric identification of proteins from silver-stained polyacrylamide gel: a method for the removal of silver ions to enhance sensitivity. Electrophoresis 1999, 20:601-605.

doi:10.1186/1477-5956-12-21

Cite this article as: Islam et al:: Proteomic analysis of Staphylococcus aureus biofilm cells grown under physiologically relevant fluid shear stress conditions. Proteome Science 2014 12:21.

\section{Submit your next manuscript to BioMed Central and take full advantage of:}

- Convenient online submission

- Thorough peer review

- No space constraints or color figure charges

- Immediate publication on acceptance

- Inclusion in PubMed, CAS, Scopus and Google Scholar

- Research which is freely available for redistribution 